\title{
Development of a pipeline for exploratory metabolic profiling of infant urine
}

Article

Published Version

Creative Commons: Attribution 4.0 (CC-BY)

Open Access

Jackson, F., Georgakopoulou, N., Kaluarachchi, M., Kyriakides, M., Andreas, N., Przysiezna, N., Hyde, M. J., Modi, N., Nicholson, J. K., Wijeyesekera, A. and Holmes, E. (2016) Development of a pipeline for exploratory metabolic profiling of infant urine. Journal of Proteome Research, 15 (9). pp. 34323440. ISSN 1535-3907 doi:

https://doi.org/10.1021/acs.jproteome.6b00234 Available at https://centaur.reading.ac.uk/83323/

It is advisable to refer to the publisher's version if you intend to cite from the work. See Guidance on citing.

To link to this article DOI: http://dx.doi.org/10.1021/acs.jproteome.6b00234

Publisher: American Chemical Society

All outputs in CentAUR are protected by Intellectual Property Rights law, including copyright law. Copyright and IPR is retained by the creators or other copyright holders. Terms and conditions for use of this material are defined in the End User Agreement.

www.reading.ac.uk/centaur 


\section{CentAUR}

Central Archive at the University of Reading

Reading's research outputs online 


\title{
Development of a Pipeline for Exploratory Metabolic Profiling of Infant Urine
}

\author{
Frances Jackson, ${ }^{\dagger}$ Nancy Georgakopoulou, ${ }^{\dagger}$ Manuja Kaluarachchi, ${ }^{\ddagger}$ Michael Kyriakides, ${ }^{\dagger}$
} Nicholas Andreas, ${ }^{\S}$ Natalia Przysiezna, ${ }^{\S}$ Matthew J. Hyde, ${ }^{\S}$ Neena Modi, ${ }^{\S}$ Jeremy K. Nicholson, ${ }^{\dagger}, \|$ Anisha Wijeyesekera, ${ }^{* \dagger}$ and Elaine Holmes ${ }^{\dagger, \|}$

${ }^{\dagger}$ Division of Computational and Systems Medicine, Department of Surgery and Cancer, Imperial College London, South Kensington Campus, London SW7 2AZ, United Kingdom

${ }^{\ddagger}$ Metabometrix Ltd, Bioincubator, Prince Consort Road, South Kensington, London SW7 2AZ, United Kingdom

${ }^{\S}$ Section of Neonatal Medicine, Department of Medicine, Imperial College London, Chelsea and Westminster Hospital Campus, London SW10 9NH, United Kingdom

"MRC-NIHR National Phenome Centre, Department of Surgery and Cancer, Imperial College London, Hammersmith Hospital Campus, London W12 0NN, United Kingdom

\section{Supporting Information}

ABSTRACT: Numerous metabolic profiling pipelines have been developed to characterize the composition of human biofluids and tissues, the vast majority of these being for studies in adults. To accommodate limited sample volume and to take into account the compositional differences between adult and infant biofluids, we developed and optimized sample handling and analytical procedures for studying urine from newborns. A robust pipeline for metabolic profiling using NMR spectroscopy was established, encompassing sample collection, preparation, spectroscopic measurement, and computational analysis. Longitudinal samples were collected from five infants from birth until 14 months of age. Methods of extraction and effects of freezing and sample dilution were assessed, and urinary contaminants from breakdown of polymers in a range of diapers and cotton wool balls were identified and compared, including propylene glycol, acrylic acid, and tert-butanol. Finally, assessment of urinary profiles obtained over the first few weeks of life revealed a dramatic change in composition, with concentrations of phenols, amino acids, and betaine altering systematically over the first few months of life. Therefore, neonatal samples require more stringent standardization of experimental design, sample handling, and analysis compared to that of adult samples to accommodate the variability and limited sample volume.

KEYWORDS: metabolic profiling, metabonomics, NMR spectroscopy, pipeline development, infant, urine

\section{INTRODUCTION}

The early life environment has been shown to exert a longreaching impact on childhood and later life health. Mode of birth, feeding, and drug exposure have all been associated with disease risk, including asthma and allergy in childhood and metabolic syndrome and cardiovascular disease in later life. ${ }^{7}$

Metabolic phenotyping of biofluids using high resolution spectroscopic techniques can be used to effectively characterize physiological or pathological processes with the aim of biomarker identification or augmenting mechanistic understanding of biology. ${ }^{1-4}$ Collection and analysis of infant biofluids imposes an analytical challenge because, in contrast to adult samples, sample volumes are typically smaller. Urine samples are collected from neonates using plastic collection bags secured to the infant with adhesive or, more commonly, disposable diapers with sterile cotton balls or pads inside the diaper, ${ }^{18-20}$ because the adhesive bags can cause skin irritation. Metabolic analysis of urine collected directly onto cotton wool or diapers can cause the introduction of contaminants from both the diaper and the cotton ball, and the sample can also be contaminated by fecal material. ${ }^{21-25,34}$

The clinical potential of metabonomics in neonatology is evidenced by a growing body of publications, demonstrating how physiological fetal development or pathological conditions affect perinatal and neonatal outcomes. ${ }^{7-10}$ In addition to providing a detailed phenotype of acute infant illnesses, metabolic profiling can provide a baseline template for understanding downstream health. For this reason, there is an increasing interest in establishing a phenotypic database of postnatal maturation and development of newborns. The literature relating to the biochemical composition of biofluids in neonates is relatively sparse with little known of the normal metabolic trajectory of the healthy neonate. ${ }^{11}$ Growth,

Received: March 17, 2016

Published: August 1, 2016 


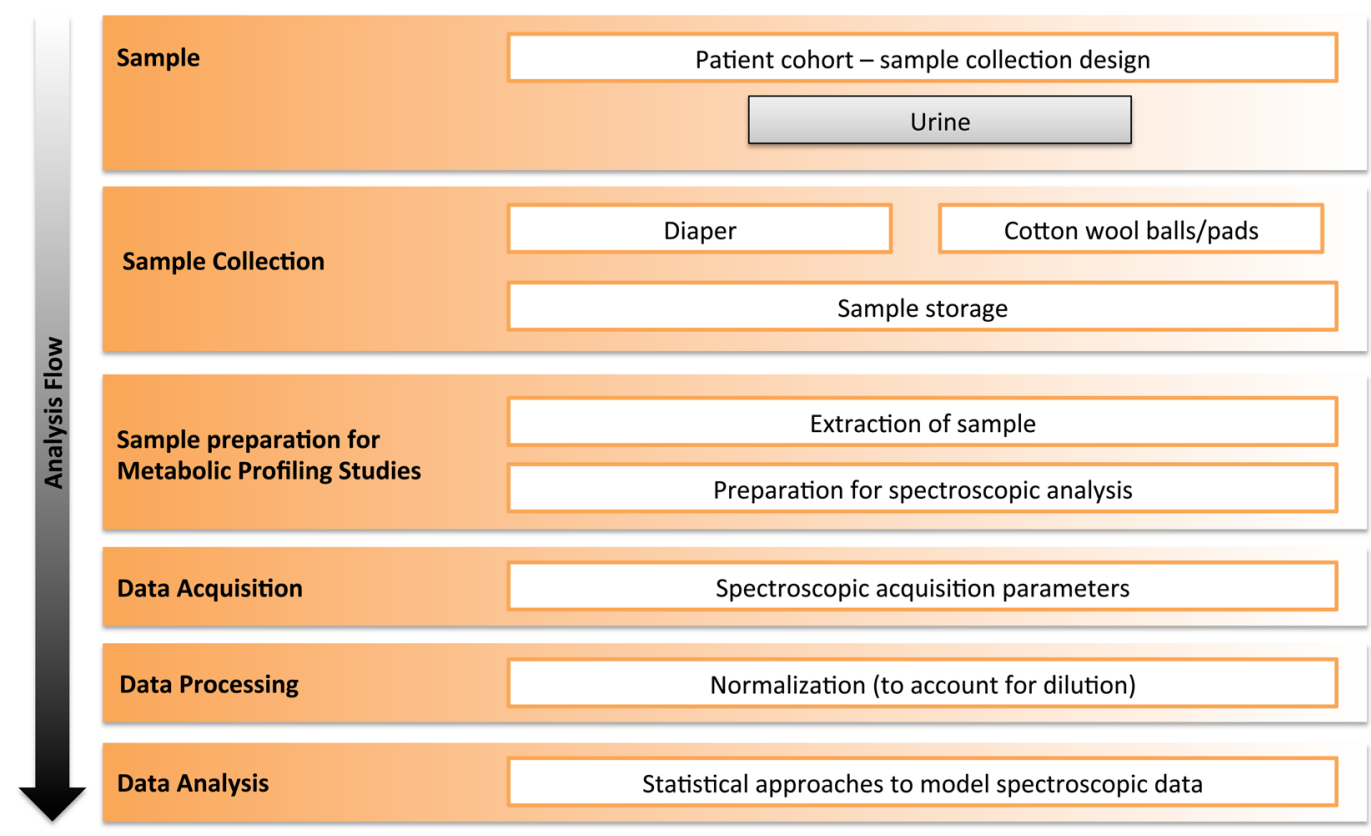

Figure 1. Schematic pipeline development for metabolic profiling of infant urine.

gestational age, nutritional composition of breast milk, and infection have been shown to influence the metabolic composition of urine and plasma. ${ }^{12-15}$

Thus far, metabonomic approaches have been used in experimental neonatology to shed light on conditions such as inborn errors of metabolism, cardiovascular, renal, and respiratory diseases, postnatal hypoxia/asphyxia, systemic infections (e.g., neonatal sepsis), brain injury, necrotizing enterocolitis, investigation of short- and long-term effects of prematurity, low birth weight, and mode of delivery as well as stratification of infants into risk groups according to response to various pharmacological treatments, such as gastroesophageal reflux treatments. However, sample collection and analysis methods have not been standardized, and the studies to date have used different sample collection procedures, analytical platforms, and data modeling strategies. ${ }^{5-7,9,11,13-17}$

We present a systematic assessment of sample collection, processing, and spectral analysis of urine samples from limited volume samples applied to newborn infants to characterize the changes in metabolic phenotype over the first few weeks of life and to address the challenge of sample limitations and potential contamination from external sources. We propose an optimized analytical pipeline for NMR spectroscopic analysis of infant urine to ensure robust data generation and to facilitate interlaboratory comparison of data sets.

\section{MATERIALS}

We developed and applied a sample collection and analysis pipeline for infant urine samples as defined in Figure 1. Urine collection was based around extraction of urine directly from cotton wool balls or onto cotton wool pads inserted into diapers because clean catch urine, which is standard for adult collection, is difficult to obtain from infants. Contaminants derived from the collection materials introduced were characterized in the urine metabolic profile. Urine samples were collected from infants born at term as part of an ongoing study at the Chelsea and Westminster Hospital. Research Ethics Committee approval was obtained from the West
London Research Ethics Committee (REC reference number $12 / \mathrm{LO} / 0203$ and 10/H0/7135)

\section{Extraction of Urine from Cotton Balls}

A volume of $3 \mathrm{~mL}$ of urine was added onto cotton wool and incubated for $2 \mathrm{~h}$ at $37^{\circ} \mathrm{C}$ (to mimic the typical infant urine collection conditions). Urine was extracted from the cotton wool in the following ways: (1) urine-filled cotton wool was frozen overnight at $-80{ }^{\circ} \mathrm{C}$ prior to extraction, (2) extracted directly from the cotton wool immediately after collection and frozen overnight at $-80{ }^{\circ} \mathrm{C}$, (3) extracted directly from the cotton wool immediately after collection, and (4) as a fresh clean catch urine sample. Extraction from the cotton wool was carried out in two ways to assess whether chemical contamination originated from exposure to laboratory equipment: (1) squeezed from the cotton wool into a falcon tube or (2) extracted into a falcon tube by centrifugation. Extraction by centrifugation involved adding the cotton wool to a falcon tube and positioning an open ended plastic tube (a cryo-tube cut into a cylindrical shape, Figure S1) underneath it to stop the cotton wool from moving to the bottom of the tube to ensure that as much urine as possible was extracted. Diapers were also processed using a similar method. Urine was added to cotton wool, and this was placed into the different diapers and incubated at $37{ }^{\circ} \mathrm{C}$ for $2 \mathrm{~h}$ after which urine was extracted by manually squeezing the cotton wool. For NMR analysis, $540 \mu \mathrm{L}$ of urine was transferred to $5 \mathrm{~mm}$ NMR tubes and $60 \mu \mathrm{L}$ of phosphate buffer (potassium dihydrogen phosphate $\left(\mathrm{KH}_{2} \mathrm{PO}_{4}\right)$, 3-trimethylsilyl propionic acid- $d_{4}$ acid sodium salt (TSP), sodium azide $\left(\mathrm{NaN}_{3}\right)$, and potassium hydroxide solution $(\mathrm{KOH}$ $45 \% \mathrm{w} / \mathrm{w})$, Sigma-Aldrich, Gilliingham, UK) was added. In addition to urine, water was used to obtain an experimental blank sample from both cotton wool and diapers to identify potential chemicals leaching from the collection materials.

Acquisition of ${ }^{1} \mathrm{H}$ NMR Spectral Profiles of Urine and Blank Samples

All ${ }^{1} \mathrm{H}$ NMR spectra were acquired on a Bruker DRX-600 spectrometer (Bruker Biospin, Karlsruhe, Germany) operating at $600.29 \mathrm{MHz}$ for proton observation using a standard one- 


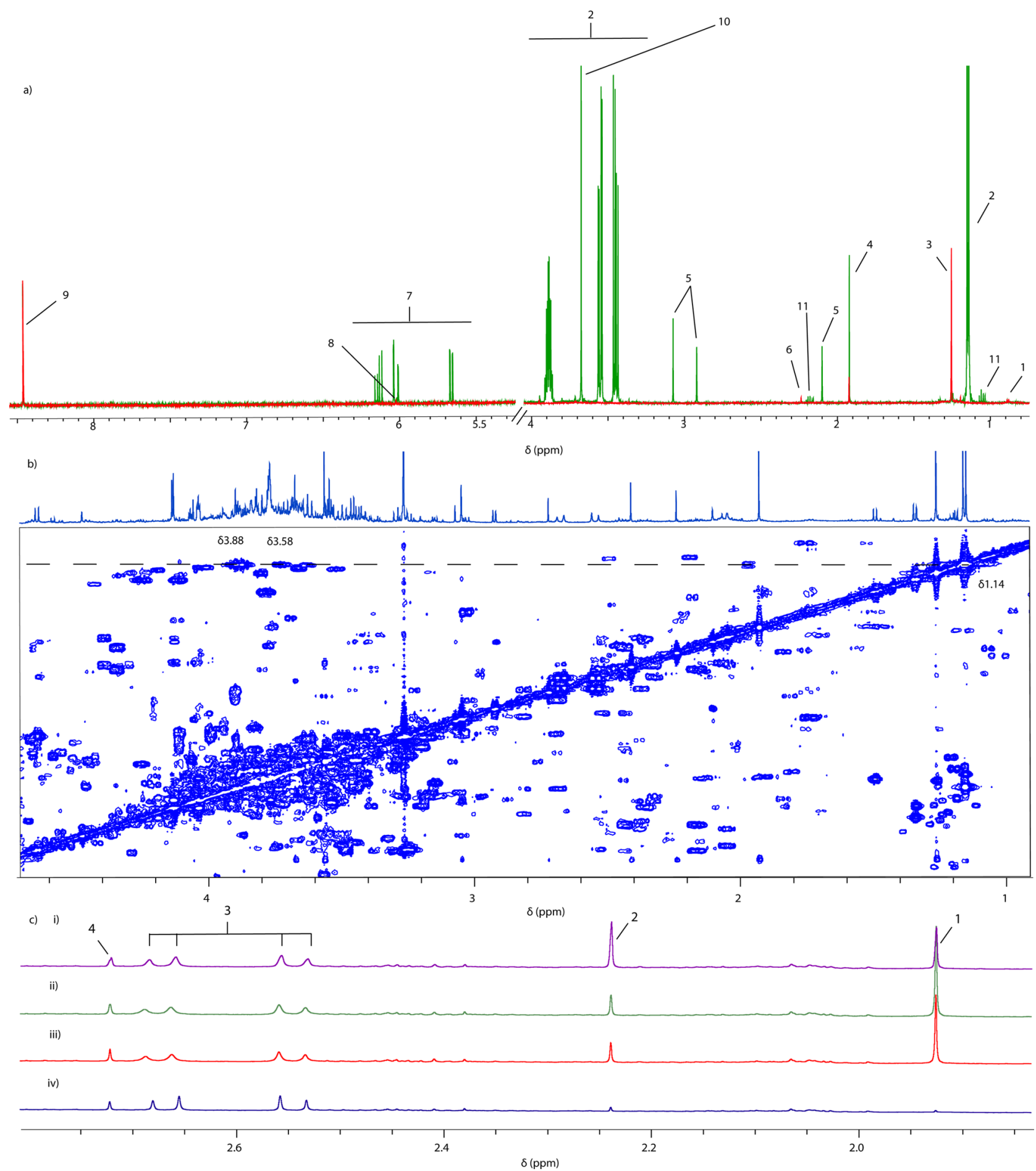

Figure 2. (a) $1 \mathrm{D}{ }^{1} \mathrm{H}$ NMR water blank spectra from cotton wool (red spectrum) and diaper blanks (green spectrum). Key: 1, isobutyl alcohol $(\delta$ $\left.0.88-\mathrm{CH}_{3}\right)$; 2, propylene glycol $\left(\delta 1.14-\mathrm{CH}_{3}, 3.43-\mathrm{CH}_{2}, 3.53-\mathrm{CH}_{2}, 3.87-\mathrm{CH}\right)$; 3, tert-butanol $\left(\delta 1.25-\mathrm{CH}_{3}\right) ; 4$, acetate $\left(\delta 1.92-\mathrm{CH}_{3}\right) ; 5$, $N, N$-dimethylacetamide $\left(\delta 2.1-\mathrm{CH}_{3}, \delta 2.9-\mathrm{CH}_{3}, \delta 3.1-\mathrm{CH}_{3}\right) ; 6$, acetone $\left(\delta 2.23-\mathrm{CH}_{3}\right) ; 7$. acrylic acid $(\delta 5.76-\mathrm{CH}, 6.02-\mathrm{CH}, 6.11-\mathrm{CH}) ; 8$, unknown metabolite $(\delta 6.02)$; 9 , formate $(\delta 8.46-\mathrm{CH})$; 10 , ethylene glycol $(\delta 3.7-\mathrm{CH})$; 11, propionate $\left(\delta 2.19-\mathrm{CH}_{2}, 1.06-\mathrm{CH}_{3}\right) .(\mathrm{b}) 2 \mathrm{D}$ $\operatorname{COSY}{ }^{1} \mathrm{H}$ NMR spectra, dotted line depicting propylene glycol $\left(\delta 1.14-\mathrm{CH}_{3}, 3.43-\mathrm{CH}_{2}, 3.87-\mathrm{CH}\right)$. (c) $1 \mathrm{D}{ }^{1} \mathrm{H}$ NMR partial urine spectra $(\delta$ 1.85-2.75) from cotton wool that was subjected to freeze-thaw cycles: (i) urine extracted from cotton wool and then subjected to one freeze thaw cycle; (ii) urine extracted from cotton wool after one freeze thaw cycle; (iii) urine extracted from cotton wool and acquired immediately; and (iv) control blank urine. Key: 1 , acetate $\left(\delta 1.92-\mathrm{CH}_{3}\right) ; 2$, acetone $\left(\delta 2.23-\mathrm{CH}_{3}\right) ; 3$, citrate $\left(\delta 2.55-\mathrm{CH}_{2}, \delta 2.66-\mathrm{CH}_{2}\right) ; 4$, dimethylamine $(\delta 2.72$ $\left.-\mathrm{CH}_{3}\right)$.

dimensional water presaturation pulse sequence [relaxation delay, $90^{\circ}, t_{1}, 90^{\circ}, t_{\mathrm{m}}, 90^{\circ}$, acquire free-induction decay (FID)]. The relaxation delay was $4 \mathrm{~s}$ followed by application of a $90^{\circ}$ radio frequency pulse; $t_{1}$ (the interpulse delay) was set to $3 \mu \mathrm{s}$, and $t_{\mathrm{m}}$ is the mixing time of $100 \mathrm{~ms}$. The probe was matched and tuned automatically to the proton transmitter resonance frequency before acquisition for each sample. Samples were run at a temperature of $300 \mathrm{~K}$. 
Spectral preprocessing and preliminary comparisons of the spectra were performed using the Bruker TopSpin 3.1 program. Multivariate statistical modeling was performed using Mathworks Matlab R2014a (The Mathworks Inc., USA) and Umetrics SIMCAP 14.0 (Umetrics, Sweden).

\section{Characterization of Chemical Contamination from Collection Materials}

For contamination of an infant urine metabolic profile to be assessed, different diapers and cotton wool brands were investigated. Three brands of diapers were assessed; for the purpose of brevity, these brands will be regarded as diaper brand 1 (Pampers), diaper brand 2 (UK hospital brand Libero), and diaper brand 3 (UK store brand Tesco). Four cotton wool brands were also assessed; three UK store brands (Tesco, Sainsbury's, and Boots) and UK hospital brand (Robinson's Healthcare). Because the capacity of urine and water as solvents may be different, the collection materials were assessed using $\mathrm{H}_{2} \mathrm{O}$ as a blank sample as well as a pooled infant urine sample from infants at postpartum ages from 0 to 19 weeks. An aliquot of $3 \mathrm{~mL}$ of a quality control infant urine sample was transferred onto four different brands of cotton wool ball (Sainsbury's, Tesco, Boots, and Robinson's Healthcare) and also onto the three different diapers (Pampers, Tesco, and Libero) combined with the cotton wool. Samples were analyzed using ${ }^{1} \mathrm{H}$ NMR spectroscopy.

Assessment of Dilution Factor and Spectral Normalization on Infant Urine Profiles

Biosamples collected from infants are often limited in amount compared with adults; this is especially true for preterm infants who in addition to producing smaller volume of urine than equivalent term babies often have other underlying conditions. Therefore, as part of the method development for infant biofluid analysis, the detection limits in terms of obtaining reproducible urine profiles were investigated for metabolic phenotyping by ${ }^{1} \mathrm{H}$ NMR spectroscopy. Urine was collected from five term-born infants in the first week postpartum and a dilution series (original concentration sample, and a dilution series of $1 / 2,1 / 4,1 / 8$ of the original concentration, as detailed in Figure S2) were created for each of the five samples. To compensate for the decrease in signal-to-noise in more dilute samples, the number of scans used to acquire spectral profiles was increased by $\sqrt{ } n$, which in turn increased the duration of the NMR experiment. The signal-to-noise ratio $(\mathrm{S} / \mathrm{N})$ of an NMR signal is improved by using signaling averaging; the $S / N$ improves with $\sqrt{n}$, where $n$ is the number of signals averaged. NMR data were normalized using the probabilistic quotient normalization (PQN), which particularly accommodates for dilution differences between samples by scaling the spectra to a median reference spectrum. ${ }^{28}$ Although the spectra of urine extracted from cotton wool or directly from diapers contained contaminants, the data were not remodeled after exclusion of these resonances because they do not dominate the coefficients of any model with the exception of the comparison of different cotton wool and diaper brands.

\section{Assessment of Age Variability on Infant Urine Profiles}

Infants develop rapidly from birth with consequential adaptation of physiology, which is likely to cause their metabolic profile to dramatically change in the first few weeks of life. Thus, unlike adults where metabolic phenotypes are generally stable over narrow age ranges, the time of sampling may be more important in infant studies. Therefore, the assessment of age variability on infant profiles was investigated for metabolic phenotyping by ${ }^{1} \mathrm{H}$ NMR spectroscopy. Urine samples taken for a mother-infant comparison were obtained from mother-infant dyads $(n=10)$ using only infants born at term. Urine samples were taken from the infant at 0,14 days, 8 weeks, and 14 months postpartum, and a further maternal sample was taken at the time of birth for comparison.

\section{RESULTS AND DISCUSSION}

\section{Assessment of Methods for Extracting Urine from Cotton Wool}

The centrifugation apparatus design (Figure S1) allowed efficient extraction of urine from the cotton wool compared to that of manual squeezing. In contrast to the study by Goodpaster and colleagues, ${ }^{25}$ who reported no chemical leaching from cotton balls, we found clear evidence of tertbutanol, isobutyl alcohol, acetone, formate, and acetate leaching into both the water blank and urine sample blank, which were extracted from the cotton. The urine sample extracted from the cotton wool after squeezing had a much higher concentration of acetone, tert-butanol, and isobutyl alcohol than the urine obtained via centrifugation, suggesting that manual squeezing of cotton wool results in greater destruction of the cotton wool fibers and therefore greater release of contaminants into the urine sample. This further underscores the need for welldefined standard operating procedures and materials, and although beyond the scope of the current study, it raises the question as to whether exposure of infants to these chemicals has any health-related consequences.

We further assessed the effect of freezing the urine sample, in the cotton wool prior to extraction as well as frozen after extraction, and compared this with urine obtained fresh from a cotton wool ball and found that there were no obvious differences in the level of chemical contamination or endogenous metabolite profile. Therefore, we find freezing the urine containing cotton wool directly after collection at -80 ${ }^{\circ} \mathrm{C}$ (which is generally more practical than performing immediate extraction of the urine) to be an acceptable practice.

Furthermore, despite the introduction of contaminants from the cotton wool, the urine profiles were highly similar, and a change in citrate concentration was the only visual difference in the endogenous compound concentration after urine had been extracted from the cotton wool, which suggests that citrate may be partially retained in the cotton wool. Furthermore, the citrate signals $\left(\delta 2.55-\mathrm{CH}_{2} ; \delta 2.66-\mathrm{CH}_{2}\right)$ became broader after treatment with cotton wool, which also suggests that there was binding of this metabolite (Figure 2(c)).

\section{Characterization of Chemical Contamination from Collection Materials}

As expected, we found that sample collection, preparation, and acquisition parameters had a strong effect on spectral composition and that the limitation of sample volume, constraints of sample collection, and the temporal variation in biofluid composition in the newborn infant introduced greater chemical variation than would typically be found in adults. Therefore, it is essential to standardize methodological protocols as much as possible. On the basis of the results of this study, we present some recommendations based on the experimental practice.

Clean catch urine is difficult to obtain from infants and thus typical collection means include placing adhesive sample collection bags on the infants or, more practically, relying on 

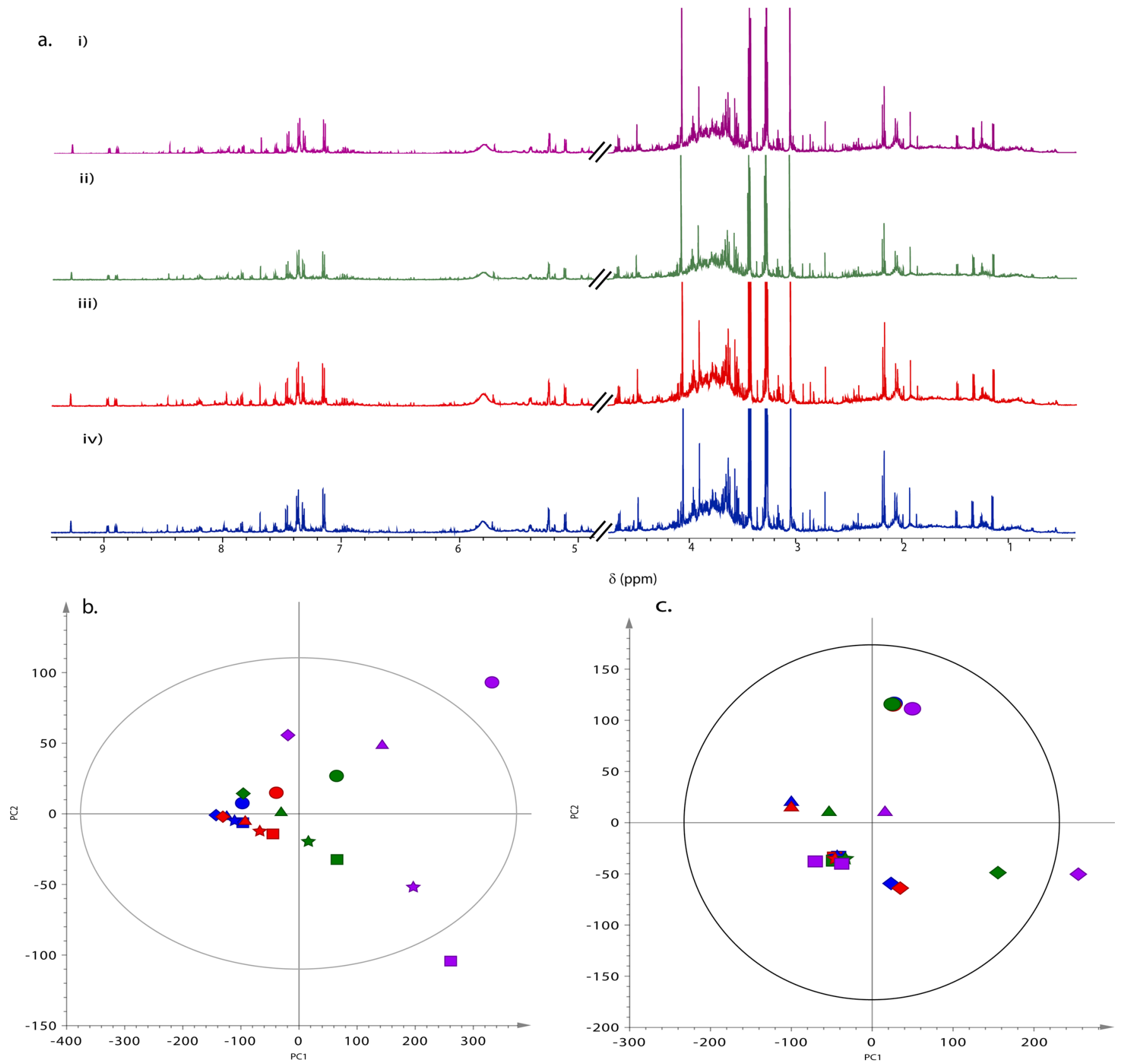

Figure 3. (a) $1 \mathrm{D}{ }^{1} \mathrm{H}$ NMR spectra from one infant with a dilution series in effect with volumes of (i) 540, (ii) 270, (iii) 135, and (iv) $68 \mu \mathrm{L}$. (b) PCA of dilution series: $R^{2}(\mathrm{X})=0.985$ (first component accounts for $84 \%$ of the total variance); $Q^{2}$ (cum.) $=0.941$. (c) PCA of normalized diluted infant samples: $R^{2}(\mathrm{X})=0.5$ (first component accounts for $32 \%$ of the total variance); $Q^{2}$ (cum.) $=0.29$. Colors represent dilution series of (blue) 540 , (red) 270, (green) 135, and (purple) $68 \mu \mathrm{L}$. Different shapes represent individual infants.

extracting urine directly from the diaper or from cotton wool balls placed inside the diaper. Both cotton wool and diapers contain chemical contaminants that contribute to the urinary metabolic profile. NMR easily detects these contaminants within the urine sample. It is therefore necessary to recognize these contaminants so that their signals can be excluded from statistical modeling, which aims to evaluate physiological or pathological processes. As expected, normalization to the median spectrum (Figure 3c) ameliorated the variation caused by differential dilution and contamination from collection material, but there is potential for a strong bias to be introduced if spectra are normalized to total intensity.

Visual comparison of the NMR spectra obtained from blank, original urine samples and cotton wool-extracted urine samples were assessed for chemical contaminants (Figure 2a). From cotton wool, major contaminants were identified as acetate $(\delta$ $\left.1.92-\mathrm{CH}_{3}\right)$, acetone $\left(\delta 2.23-\mathrm{H}_{3}\right)$, formate $(\delta 8.46-\mathrm{CH})$, citrate $\left(\delta 2.55-\mathrm{CH}_{2}, 2.66-\mathrm{CH}_{2}\right)$, propylene glycol $(\delta 1.14$ $\left.-\mathrm{CH}_{3}, 3.43-\mathrm{CH}, 3.54-\mathrm{CH}, 3.87-\mathrm{CH}_{2}\right)$, tert-butanol $(\delta 1.25$ $\left.-\mathrm{CH}_{3}\right)$, and isobutyl alcohol $\left(\delta 0.88-\mathrm{CH}_{3}\right)$ as well as an as yet unidentified peak at $\delta 6.02$.

Different cotton wool brands contained variable concentrations of these metabolites, but the qualitative presence of the metabolites was consistent. Contaminants from diapers included propylene glycol $\left(\delta 1.14-\mathrm{CH}_{3}, 3.4-\mathrm{CH}, 3.6\right.$ $\left.-\mathrm{CH}, 3.9-\mathrm{CH}_{2}\right), N, N$-dimethylacetamide $\left(\delta 2.1-\mathrm{CH}_{3}, 2.9\right.$ $\left.-\mathrm{CH}_{3}, 3.1-\mathrm{CH}_{3}\right)$, ethylene glycol $(\delta 3.7-\mathrm{CH})$, tert-butanol $\left(\delta 1.25-\mathrm{CH}_{3}\right)$, and isobutyl alcohol $\left(\delta 0.88-\mathrm{CH}_{3}\right)$ as well as 


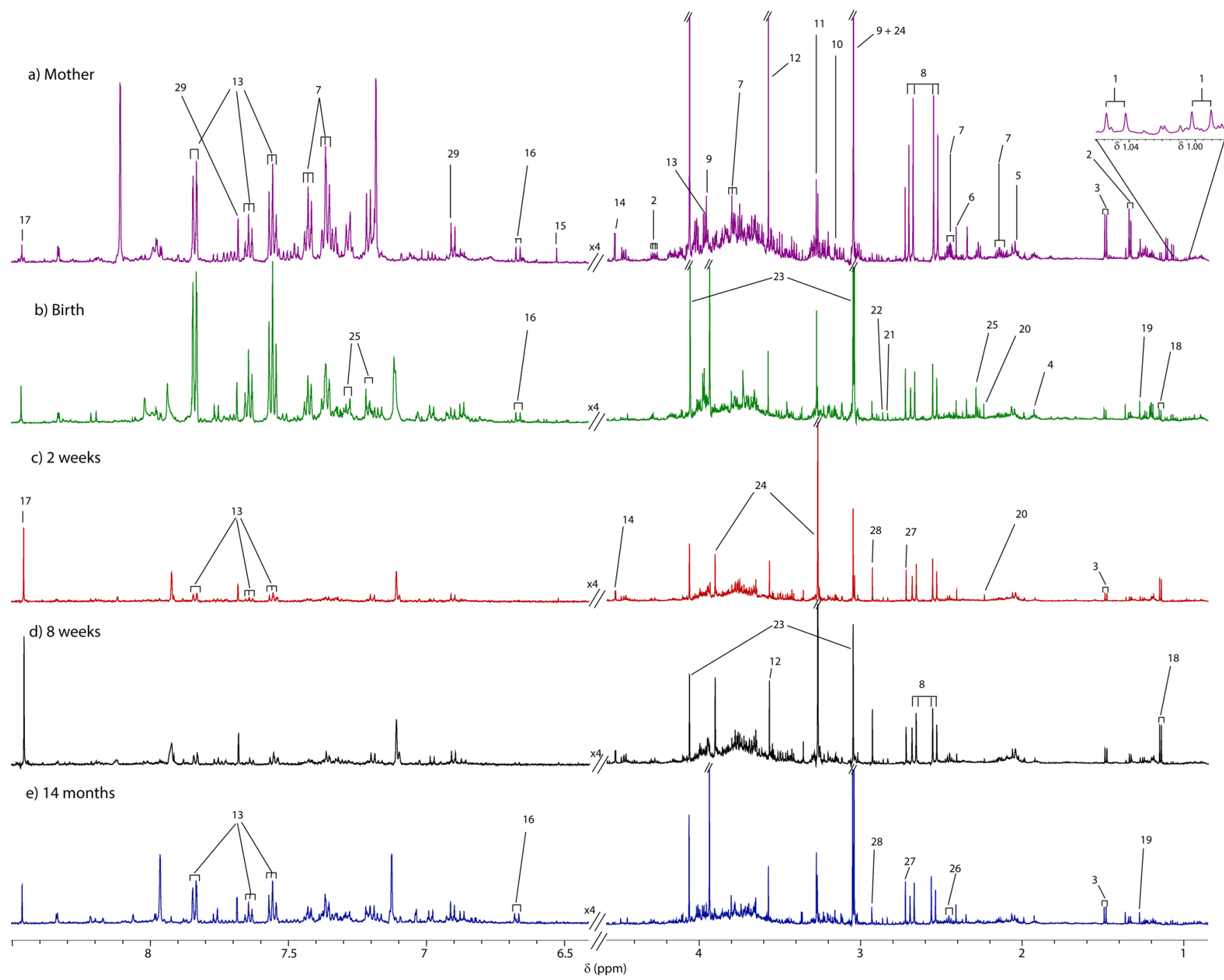

Figure 4. Mother-baby urine-matched $1 \mathrm{D}{ }^{1} \mathrm{H}$ NMR spectra showing the age variability from birth to 14 months postpartum for (a) mother, (b) birth, (c) 2 weeks, (d) 8 weeks, and (e) 14 months. Key: 1 , valine $\left(\delta 0.99-\mathrm{CH}_{3}, 1.04-\mathrm{CH}_{3}\right) ; 2$, lactate $(\delta 1.33-\mathrm{CH}, 4.11-\mathrm{CH}) ; 3$, alanine $(\delta$ $\left.1.47-\mathrm{CH}_{3}\right) ; 4$, acetate $\left(\delta 1.92-\mathrm{CH}_{3}\right) ; 5$, uromodulin $\left(\delta 2.06-\mathrm{CH}_{2}\right) ; 6$, succinate $\left(\delta 2.42-\mathrm{CH}_{2}\right) ; 7$, phenylacetylglutamine $(\mathrm{PAG})\left(\delta 2.14-\mathrm{CH}_{2}\right.$, $2.46-\mathrm{CH}_{2}, 3.77-\mathrm{CH}_{2}, 7.35(-\mathrm{CH})_{3} ; 8$, citrate $\left(\delta 2.55-\mathrm{CH}_{2}, 2.66-\mathrm{CH}\right) ; 9$, creatine $\left(\delta 3.02-\mathrm{CH}_{3}\right.$ (overlapped with creatinine), $\left.3.92-\mathrm{CH}_{2}\right)$; 10 , proline betaine $\left(\delta 3.11-\mathrm{CH}_{3}, 3.30-\mathrm{CH}_{3}\right) ; 11$, trimethylamine- $\mathrm{N}$-oxide $\left(\delta 3.25-\mathrm{CH}_{3}\right) ; 12$, glycine $\left(\delta 3.55-\mathrm{CH}_{2}\right) ; 13$, hippurate $(\delta 3.95$ $-\mathrm{CH}) ; 14, N$-methylnicotinamide $(\delta 4.48-\mathrm{CH}) ; 15$, fumarate $\left(\delta 6.53-\mathrm{CH}_{3}\right) ; 16$, urocanic acid $(\delta 6.65)$; 17 , formate $(\delta 8.45-\mathrm{CH}) ; 18$, propylene glycol $\left(\delta 1.14-\mathrm{CH}_{3}\right) ; 19$, tert-butanol $\left(\delta 1.25-\mathrm{CH}_{3}\right) ; 20$, acetone $\left(\delta 2.24-\mathrm{CH}_{3}\right) ; 21$, methylguanidine (tentative assignment; $\left.\delta 2.23-\mathrm{CH}_{3}\right) ; 22$, trimethylamine $\left(\delta 2.28-\mathrm{CH}_{3}\right) ; 23$, creatinine $\left(\delta 3.05-\mathrm{CH}_{3}, 4.06-\mathrm{CH}_{2}\right) ; 24$, betaine $\left(\delta 3.25\left(-\mathrm{CH}_{3}\right), 3.89-\mathrm{CH}_{2}\right) ; 25$, 4-cresol sulfate $(\delta$ 2.22 $\left.-\mathrm{CH}_{3}, 7.11-\mathrm{CH}, 7.18-\mathrm{CH}\right) ; 26$, 2-oxoglutarate $(\delta 2.42-\mathrm{CH})$; 27, dimethylamine $\left(\delta 2.72-\mathrm{CH}_{3}\right)$; 28, dimethylglycine $\left(\delta 2.93-\mathrm{CH}_{3}\right)$; and $29,3-$ methyl-histidine $(\delta 6.93-\mathrm{CH}, 7.69-\mathrm{CH})$.

acrylic acid $\left(\delta 5.76-\mathrm{CH}_{2}, 6.02-\mathrm{CH}_{2}, 6.11-\mathrm{CH}\right)$ and propionate $\left(\delta 2.19-\mathrm{CH}_{2}, 1.06-\mathrm{CH}_{3}\right)$. A diaper's absorbent core is predominantly derived from polymers made from very small particles of acrylic acid derivatives, giving it its highly absorbent properties. Our results would suggest that the observed contaminants are introduced either during the manufacturing process or that degradation of this polymer may have occurred, which would argue for further investigation of the effect of shelf life or storage on the chemicals that leach from diapers.

Although some of these contaminants, such as polyethylene glycol in diapers ${ }^{25}$ and propylene glycol in infant urine, have been previously reported and associated with baby wipes, ${ }^{34}$ to our knowledge we provide here the most comprehensive assignment of exogenous chemicals deriving from collection procedures. A systematic analysis of the effect of different diapers on synthetic urine by Goodpaster and colleagues showed that multivariate analysis of either NMR or LC-MS spectra resulted in clustering of spectra according to the brand of diaper, which is consistent with our results (Figure S3). In Goodpaster's study, it was estimated that up to $59 \%$ of the NMR spectrum was influenced by chemical contamination from diapers. Thus, it is important that the brand of diaper is standardized across a study to prevent the introduction of extraneous variation.

Assessment of Dilution Factor and Spectral Normalization on Infant Urine Profiles

Because urine sample dilution can vary greatly, the systematic effect of dilution on the spectral profile was assessed. The NMR spectra obtained from an individual sample with each spectrum 
corresponding to a different dilution factor, is shown in Figure 3. These results indicate that, after adjustment for the number of scans to account for differences in signal-to-noise, ${ }^{30}$ there was little visual effect of dilution on the NMR spectral signal intensities (Figure 3a). However, the dilution factor (indicated by color) was shown to affect the statistical models obtained when non-normalized spectra were used, suggesting that dilution has a physicochemical effect on the observation of certain metabolite signals, and metabolic differences between individuals were more easily observed in the more dilute samples (indicated by symbols, Figure $3 b$ ). Once normalization was applied to the spectral data, which is typical practice for metabolic profiling studies, using either PQN normalization with reference to the median spectrum (Figure 3c) or noise (data not shown) as a standardizing factor, the effect of dilution was reduced, and clear interindividual differences between the infants became apparent at all dilution factors with the exception of two infants whose profiles were largely superimposed in the first two components (as indicated by the squares and stars in Figure 3c). This indicates that standard normalization procedures can largely resolve the effect of dilution on the spectroscopic profiles. For one infant (represented by diamond symbols), the effect of urinary dilution was still clearly visible; this infant had the lowest creatinine to noise ratio, suggesting that the urine sample from this individual was the most dilute to begin with. A more accurate way of controlling for differential concentrations between samples would be to ensure that all samples were adjusted for osmolality prior to analysis, but this would require extra sample and would introduce an extra time constraint and potential for human error. Because for three of the five infants the two most dilute samples were situated to the left of the corresponding cluster for that individual, we assumed that an appropriate dilution factor to employ would be no more than double the original sample volume.

\section{Age Variability in Infant Urine Profiles}

Given the generally chaotic start to life, it is often difficult to standardize the exact time of biofluid sampling in infants. However, this and many previous studies highlight the high variability in neonatal urine composition in relation to age. Over the first few weeks of life, infant metabolism undergoes rapid changes, and this is reflected in the dynamic urine profiles. Here, we show that urinary NMR spectral profiles of infants at the time of birth (Figure $4 b$ ) are evidently very similar to urinary samples collected from the mother at the same time (Figure 4a). Throughout pregnancy, the developing fetus relies on the mother for nutrient uptake, waste removal, and gas exchange, which occurs through the placenta; thus, similarities in the global urinary metabolic profile are observed. $^{32,33}$ The similarities between mother and newborn are particularly apparent in the aromatic region of the NMR spectra. A higher concentration of hippurate and phenylacetylglutamine (PAG) in the infant birth sample can be seen in comparison to that at later time points. We would expect to see low concentrations of phenolic and aromatic compounds in infant urine because these molecules are mainly produced by gut microbiota. We know from the microbiome literature and from studies in rodent models that colonization of the infant gut largely begins at birth, and that over a period of time as the symbiotic gut bacteria evolve, there is an increase in molecules such as hippurate, PAG (or the equivalent glycine conjugate in rodents), indoles, and methylamines. ${ }^{31}$ However, many papers report an absence of signals in the spectral window of infant urine where phenolic and other aromatic molecules would be expected to arise. In the urine sample obtained immediately postpartum, hippurate, 4-cresylsulfate, and PAG are found in the infant urine in approximately equivalent concentrations to the maternal urine. Although there are strong similarities between mother and newborn infant urine, some differences between maternal and infant urine composition are visible at this initial sampling point, including the presence of higher concentrations of trimethylamine and formate and lower concentrations of methylhistidine.

After 2 weeks, the infant's urine spectrum has become more dilute compared to that of the mother's urine (Figure 4c). The most striking difference between these spectra is that there is much less creatinine and almost no hippurate with a reduction in the excretion of most aromatic compounds in the week 2 sample from the infant compared to that of the birth sample. This reinforces the observation of products of mother-fetal exchange of nutrients and waste products in the infant urine samples obtained immediately postpartum. At this time, betaine is also found in higher concentrations in the neonatal urine compared to that in the maternal or later urine samples from the baby and is thought to reflect the as yet immature kidneys. ${ }^{37}$

At 14 months (Figure 4d), the infant will have grown considerably, will be eating more solid foods, and will have a larger community of bacteria in their gut, which contributes to metabolism and therefore their urine metabolic profile. ${ }^{35}$ The NMR spectra measured at 14 months showed higher concentrations of creatinine, which is related to muscle mass, as well as higher levels of metabolites found in the aromatic region (e.g., hippurate and PAG), which are cometabolites produced from gut microorganisms. ${ }^{36}$

\section{Relevance of Results to Neonatal Research}

Currently, most clinical tests available in neonatology rely on technologies that can only measure a single chemical in blood, urine, or other biofluids; these tests are neither sensitive nor specific for any particular disease. ${ }^{26-28}$ Analysis of the infant urinary metabolome offers a holistic approach to systems medicine with the promise to enhance clinical chemistry diagnostics in several pathologic conditions related to newborns and significantly improve monitoring of physiological development and disease. ${ }^{29}$ The goal is for each condition or disease to derive a discrete metabolic phenotype, comprised essentially from small but significant differences in certain metabolites. ${ }^{10}$ Thus, scientists need to be sure that these discriminant metabolites are products of a neonate's metabolism and kidney function and not just byproducts of the cotton wool and diapers used to collect the biofluids.

This issue of possible contaminants has been examined and further reported using NMR-based metabonomics in three diaper brands using the "diaper plus cotton ball" technique. ${ }^{30}$ The authors found that the NMR spectra of the two cotton ball brands examined were identical; thus, only one brand of cotton ball was used for "diaper plus cotton ball" sample analysis. It also indicated that, although certain diaper brands are more likely to exhibit distinct contaminant profiles than others, the contamination levels of newborn urine collected using the "diaper plus cotton ball" technique generally contain less diaper contamination than the "diaper alone" technique. Therefore, it is advised that diapers and cotton balls are chemically characterized using metabonomic methodologies prior to 
Table 1. Recommendations

\begin{tabular}{|c|c|}
\hline factor & recommendation \\
\hline \multirow[t]{3}{*}{$\begin{array}{l}\text { optimize recovery of urine } \\
\text { volumes }\end{array}$} & use cotton wool in diapers to collect urine \\
\hline & $\begin{array}{l}\text { include water and urine blank samples in analytical run and apply database of known contaminants to identify chemical } \\
\text { contaminants arising from diaper or cotton wool }\end{array}$ \\
\hline & use modified centrifugation technique for extraction \\
\hline \multirow{2}{*}{$\begin{array}{l}\text { minimization of chemical } \\
\text { contaminants }\end{array}$} & use same brand of collection kit (cotton wool, diapers) \\
\hline & use centrifugation to minimize extraction of contaminants and maximize volume \\
\hline \multirow{2}{*}{$\begin{array}{l}\text { optimization of sampling } \\
\text { handling }\end{array}$} & use only one freeze-thaw cycle \\
\hline & establish clear SOP for biofluid collection and preparation \\
\hline \multirow{2}{*}{$\begin{array}{l}\text { optimization of spectral } \\
\text { preprocessing }\end{array}$} & normalize spectral data to the median spectrum \\
\hline & remove spectral features corresponding to known chemical contaminants prior to modeling the data \\
\hline age variability & $\begin{array}{l}\text { detailed records/metadata relating to demographic factors including age, post menstrual age, and feeding } \\
\text { when possible, impose a narrow age range inclusion criteria }\end{array}$ \\
\hline
\end{tabular}

analysis of study urine samples. ${ }^{22}$ In this way, contaminants arising from collection material would be manageable, and unwanted variation introduced by contamination can be minimized.

Infant age is an important variable that contributes to the metabolic profile of biofluids, and thus, establishing the metabolic profiles linked to age has become a priority. From birth, the infant urinary metabolic profile can be related to the mother and changes dramatically in the first few days after birth. The infant's body continues to develop outside of the womb, and as they start to establish a gut microbiome, the metabolic profile reiterates this through changes in their metabolome; this is particularly evident over the first few days of life. The knowledge of an age-related metabolic profile can make the interpretation of data for other effects such as disease easier, which is important in biomarker discovery for disease diagnosis or prognosis. In studies where age is not standardized, this should be appreciated and incorporated into the data modeling strategy.

Standardization of experimental design and subsequent analysis is imperative for interpretation of metabolic data generated in neonatal studies. Improved tools for metabonomic analysis incorporating a reproducible analytical pipeline for sample collection, processing, and analysis along with its integration with other '-omics' (proteomics, epigenomics, etc.) analysis will make this technology valuable in the clinical setting, opening new possibilities for disease diagnosis and assessment of disease risk. In particular, metabolic profiling of neonates will not only impact diagnosis and treatment of neonatal diseases but may deliver validated biomarkers of early life stress, which could be used for predicting individual predisposition to disease or to the monitoring of disease progression and therapeutic management (Table 1).

\section{ASSOCIATED CONTENT}

\section{S Supporting Information}

The Supporting Information is available free of charge on the ACS Publications website at DOI: 10.1021/acs.jproteome.6b00234.

S1, schematic of urine extraction; S2, urine dilution series and $1 \mathrm{D}{ }^{1} \mathrm{H}$ NMR experimental design; S3, PCA scores scatter plot showing cotton ball and diaper contamination urine profiles analyzed by $1 \mathrm{D}{ }^{1} \mathrm{H}$ NMR spectroscopy; S4, orthogonal projections to latent structures discriminant analysis loading coefficients plot showing discriminatory metabolites separating between cotton wool balls that were squeezed vs centrifuged (PDF)

\section{AUTHOR INFORMATION}

\section{Corresponding Author}

*E-mail: anisha.wijeyesekera04@imperial.ac.uk. Tel: +44 (0)20 75943107.

\section{Notes}

The authors declare no competing financial interest.

\section{ACKNOWLEDGMENTS}

The authors thank the Imperial-National Institute for Health Research (NIHR) Clinical Phenome Centre for support. The centre is supported by the NIHR Imperial Biomedical Research Centre based at Imperial College Healthcare National Health Service (NHS) Trust and Imperial College London. The views expressed are those of the author(s) and not necessarily those of the NHS, the NIHR, or the Department of Health. F.J. acknowledges the Imperial College Stratified Medicine Graduate Training Program in Systems Medicine and Spectroscopic Profiling (STRATiGRAD) for financial support and scientific contribution. The authors would also like to thank the MRC-NIHR National Phenome Centre (NPC), which is supported by the UK Medical Research Council (MRC) [in association with National Institute for Health Research (NIHR) (England)] Grant MC PC 12025. The financial support of Bruker Biospin, Waters Corporation, Metabometrix, and Imperial College is also gratefully acknowledged by the NPC.

\section{ABBREVIATIONS}

NMR, nuclear magnetic resonance; PCA, principal components analysis; OPLS-DA, orthogonal projections to latent structures discriminant analysis; $\mathrm{PQN}$, probabilistic quotient normalization; FID, free induction decay; TSP, 3-trimethylsilyl propionic acid- $d_{4}$ acid sodium salt; PAG, phenylacetylglutamine

\section{REFERENCES}

(1) Nicholson, J. K.; Lindon, J. C. Systems biology: Metabonomics. Nature 2008, 455 (7216), 1054-6. 
(2) Nicholson, J. K.; Connelly, J.; Lindon, J. C.; Holmes, E. Metabonomics: a platform for studying drug toxicity and gene function. Nat. Rev. Drug Discovery 2002, 1 (2), 153-61.

(3) Fiehn, O. Metabolomics-the link between genotypes and phenotypes. Plant Mol. Biol. 2002, 48 (1-2), 155-71.

(4) Holmes, E.; Wilson, I. D.; Nicholson, J. K. Metabolic phenotyping in health and disease. Cell 2008, 134 (5), 714-7.

(5) Graça, G.; Goodfellow, B. J.; Barros, A. S.; et al. UPLC-MS metabolic profiling of second trimester amniotic fluid and maternal urine and comparison with NMR spectral profiling for the identification of pregnancy disorder biomarkers. Mol. BioSyst. 2012, 8 (4), 1243-54.

(6) Lindon, J. C.; Holmes, E.; Nicholson, J. K. Pattern recognition methods and applications in biomedical magnetic resonance. Prog. Nucl. Magn. Reson. Spectrosc. 2001, 39 (1), 1-40.

(7) Fanos, V.; Antonucci, R.; Barberini, L.; Noto, A.; Atzori, L. Clinical application of metabolomics in neonatology. J. Matern.-Fetal Neonat. Med. 2012, 25 (Suppl1), 104-9.

(8) Fanos, V.; Antonucci, R.; Barberini, L.; Atzori, L. Urinary metabolomics in newborns and infants. Adv. Clin. Chem. 2012, 58, 193-223.

(9) Fanos, V.; Antonucci, R.; Atzori, L. Metabolomics in the developing infant. Curr. Opin. Pediatr. 2013, 25 (5), 604-11.

(10) Mussap, M.; Noto, A.; Cibecchini, F.; Fanos, V. The importance of biomarkers in neonatology. Semin Fetal Neonatal Med. 2013, 18 (1), $56-64$.

(11) Fanos, V.; Van den Anker, J.; Noto, A.; Mussap, M.; Atzori, L. Metabolomics in neonatology: fact or fiction? Semin Fetal Neonatal Med. 2013, 18 (1), 3-12.

(12) Trump, S.; Laudi, S.; Unruh, N.; Goelz, R.; Leibfritz, D. 1HNMR metabolic profiling of human neonatal urine. MAGMA 2007, 19 (6), 305-12.

(13) Moltu, S. J.; Sachse, D.; Blakstad, E. W.; et al. Urinary metabolite profiles in premature infants show early postnatal metabolic adaptation and maturation. Nutrients 2014, 6 (5), 1913-30.

(14) Gu, H.; Pan, Z.; Xi, B.; et al. 1 H NMR metabolomics study of age profiling in children. NMR Biomed. 2009, 22 (8), 826-833.

(15) Foxall, P. J.; Bewley, S.; Neild, G. H.; Rodeck, C. H.; Nicholson, J. K. Analysis of fetal and neonatal urine using proton nuclear magnetic resonance spectroscopy. Arch Dis Child - Fetal Neonatal Ed. 1995, 73 (3), F153-F157.

(16) Romero, R.; Espinoza, J.; Kusanovic, J. P.; et al. The preterm parturition syndrome. BJOG 2006, 113 (Suppl), 17-42.

(17) Dessì, A.; Atzori, L.; Noto, A.; et al. Metabolomics in newborns with intrauterine growth retardation (IUGR): urine reveals markers of metabolic syndrome. J. Matern.-Fetal Neonat. Med. 2011, 24 (Suppl 2), 35-9.

(18) Atzori, L.; Antonucci, R.; Barberini, L.; et al. 1H NMR-based metabolomic analysis of urine from preterm and term neonates. Front. Biosci., Elite Ed. 2011, 3, 1005-12.

(19) Fanos, V.; Caboni, P.; Corsello, G.; et al. Urinary (1)H-NMR and GC-MS metabolomics predicts early and late onset neonatal sepsis. Early Hum. Dev. 2014, 90 (Suppl 1), S78-83.

(20) Fanos, V.; Barberini, L.; Antonucci, R.; Atzori, L. Pharmametabolomics in neonatology: is it a dream or a fact? Curr. Pharm. Des. 2012, 18 (21), 2996-3006.

(21) Hutton, N. M.; Schreiner, R. L. Urine Collection in the Neonate. J. Obstet Gynecol \& Neonatal Nurs. 1980, 9 (3), 165-169.

(22) Farrell, M.; Devine, K.; Lancaster, G.; Judd, B. A method comparison study to assess the reliability of urine collection pads as a means of obtaining urine specimens from non-toilet-trained children for microbiological examination. J. Adv. Nurs. 2002, 37 (4), 387-93.

(23) Seifert, J. A.; Ross, C. A.; Deutsch, J. C.; Awad, J. A.; Norris, J. M. Validating methods of urine collection: a pilot study. Urol Nurs. 2002, 22 (2), 113-7.

(24) Lee, E. J.; Arbuckle, T. E. Urine-sampling methods for environmental chemicals in infants and young children. J. Exposure Sci. Environ. Epidemiol. 2009, 19 (7), 625-33.
(25) Goodpaster, A. M.; Ramadas, E. H.; Kennedy, M. A. Potential effect of diaper and cotton ball contamination on NMR- and LC/MSbased metabonomics studies of urine from newborn babies. Anal. Chem. 2011, 83 (3), 896-902.

(26) Zohouri, F. V.; Swinbank, C. M.; Maguire, A.; Moynihan, P. J. Is the fluoride/creatinine ratio of a spot urine sample indicative of 24-h urinary fluoride? Community Dent Oral Epidemiol. 2006, 34 (2), 1308.

(27) Gika, H. G.; Theodoridis, G. A.; Wingate, J. E.; Wilson, I. D. Within-day reproducibility of an HPLC-MS-based method for metabonomic analysis: application to human urine. J. Proteome Res. 2007, 6 (8), 3291-303.

(28) Dieterle, F.; Ross, A.; Schlotterbeck, G.; Senn, H. Probabilistic quotient normalization as robust method to account for dilution of complex biological mixtures. Application in $1 \mathrm{H}$ NMR metabonomics. Anal. Chem. 2006, 78 (13), 4281-90.

(29) Dona, A. C.; Jiménez, B.; Schäfer, H.; et al. Precision highthroughput proton NMR spectroscopy of human urine, serum, and plasma for large-scale metabolic phenotyping. Anal. Chem. 2014, 86 (19), 9887-94.

(30) Spagou, K.; Wilson, I. D.; Masson, P.; et al. HILIC-UPLC-MS for exploratory urinary metabolic profiling in toxicological studies. Anal. Chem. 2011, 83 (1), 382-90.

(31) Holmes, E.; Li, J. V.; Athanasiou, T.; Ashrafian, H.; Nicholson, J. $\mathrm{K}$. Understanding the role of gut microbiome-host metabolic signal disruption in health and disease. Trends Microbiol. 2011, 19 (7), 34959.

(32) Lager, S.; Powell, T. L. Regulation of nutrient transport across the placenta. J. Pregnancy 2012, 2012, 179827.

(33) Tea, I.; Le Gall, G.; Küster, A.; et al. 1H-NMR-based metabolic profiling of maternal and umbilical cord blood indicates altered materno-foetal nutrient exchange in preterm infants. PLoS One 2012, 7 (1), e29947.

(34) O'Sullivan, A.; He, X.; McNiven, E.; et al. Metabolomic Phenotyping Validates the Infant Rhesus Monkey as a Model of Human Infant Metabolism. J. Pediatr. Gastroenterol. Nutr. 2013, 56, $355-363$.

(35) Want, E. J.; Wilson, I. D.; Gika, H.; et al. Global metabolic profiling procedures for urine using UPLC-MS. Nat. Protoc. 2010, 5 (6), 1005-18.

(36) Palmer, C.; Bik, E. M.; DiGiulio, D. B.; et al. Development of the Human Infant Intestinal Microbiota. PLoS Biol. 2007, 5 (7), e177.

(37) Davies, S. E. C.; Woolf, D. A.; Chalmers, R. A.; et al. Proton nmr studies of betaine excretion in the human neonate: consequences for choline and methyl group supply. J. Nutr. Biochem. 1992, 3, 523-530. 